\title{
Track Initialization from Incomplete Measurements
}

\author{
Martina Daun ${ }^{1}$, Christian R. Berger ${ }^{2}$, Wolfgang Koch $^{1}$ \\ ${ }^{1}$ SDF (Sensor Networks and Data Fusion), FGAN e.V. \\ \{daun|w.koch\}@fgan.de \\ ${ }^{2}$ University of Connecticut, Dept. of Electrical and Computer Engineering \\ crberger@engr.uconn.edu
}

\begin{abstract}
Target tracking from incomplete measurements using Extended Kalman filtering suffers from unstable behavior due to difficult initialization. Instead of using numerical batch-estimators, we offer an analytical approach to initialize the filter from a minimum number of observations. Additionally, we provide the possibility to estimate only sub-sets of parameters, and to reliably model resulting added uncertainties by the covariance matrix.

In a practical example with 3D bearings-only measurements, we implement our approach using geometrical considerations and give numerical results. The Monte-Carlo simulations show that the estimation accuracy is close to the Cramer-Rao lower bound and that the initialization is consistent according to the chi-square $95 \%$ acceptance region within a $40 \mathrm{~km}$ by $40 \mathrm{~km}$ area, which is four times the distance between the two sensors.
\end{abstract}

\section{Introduction}

Tracking of targets using measurements in polar or spherical coordinates and modeling their movement in Cartesian coordinates is a typical scenario [BSLK01, vK91]. The common approach utilizes generalized Kalman filtering, which can also work on incomplete measurements, e.g., only azimuth/elevation or azimuth/range in three spatial dimensions, provided certain observability conditions are satisfied [Bec00]. Especially in the case of incomplete measurements, the initialization of the extended Kalman filter with a state estimate and corresponding state covariance is crucial for its performance.

In the case of incomplete measurements this can not be accomplished by direct inversion of the measurement function. Multiple measurements will have to be combined for initialization, which calls for a sensible data fusion.

Typically, numerical optimization is used to find a Maximum Likelihood (ML) estimate [KBSL01], needing a large number of measurements. We offer instead an analytical approach using a minimum number of measurements, which returns an initial estimate and a corresponding covariance. By making statistical assumptions about some components of the state vector, we can initialize these state elements with their mean and covariance, and thereby reduce the number of unknowns to be estimated. Accordingly, the number of measurements to be used for initializing is farther reduced; more importantly, we will derive the added uncertainty as part of the covariance matrix. 


\section{System Model}

Let $\mathbf{x}$ be the state vector of the target with dimension $\eta_{\mathbf{x}}$ (usually in Cartesian coordinates). The measurement function $h$ is generally non-linear, dependent on the observer position $\mathbf{x}_{s}$ and not invertible, i.e. $\mathbf{z}=h\left(\mathbf{x}-\mathbf{x}_{s}\right)+\mathbf{w}$. The zero mean, Gaussian measurement noise $\mathbf{w}$ and the observation $\mathbf{z} \epsilon M$ are of dimension $\eta_{\mathbf{z}}$.

We partition the state vector into two subvectors $\mathbf{x}_{o}$ and $\overline{\mathbf{x}}_{o}$, one which is initially estimated by a function of the first measurements and the other which is initialized by appropriate modeling assumptions. W.l.o.g., we can reorder the elements of $\mathbf{x}$ as $\left[\mathbf{x}_{o}, \overline{\mathbf{x}}_{o}\right]^{\prime}$, which we can formally express as

$$
\mathbf{x}=K \mathbf{x}_{o}+\bar{K} \overline{\mathbf{x}}_{o}
$$

with $K=[I, 0]^{\prime}$ and $\bar{K}=[0, I]^{\prime}$ being of appropriate dimensions $\eta_{\mathbf{x}} \times \eta_{\mathbf{x}_{o}}$ and $\eta_{\mathbf{x}} \times \eta_{\overline{\mathbf{x}}_{o}}$. Using $k$ diffrent measurements we try to find a function $t$ with $t: M^{k} \rightarrow \mathbb{R}^{\eta_{\mathbf{x}_{o}}}$ which fulfills the following condition.

$$
\text { if } \quad \bar{x}_{o}=E\left[\bar{x}_{o}\right] \text { then } t \circ h(\mathbf{x})=\mathbf{x}_{o}
$$

i.e., if our assumptions on $\overline{\mathbf{x}}_{o}$ hold, then $t$ gives the correct estimate. In the case of linear functions, this would be equivalent to an unbiased estimator.

We have to combine at least $k$ measurements so that $k \eta_{\mathbf{z}} \geq \eta_{\mathbf{x}_{o}}$. To simplify notation, we will use the notation $t(\mathbf{z})$ also when referring to $t\left(\mathbf{z}^{k}\right)$ and use $h(\mathbf{x})$ even for mapping to $\mathbf{z}^{k}$. Since the function $t$ is generally not readily available, defining it in a sensible way will be one of the main tasks of this work.

Let the likelihood function $p(\mathbf{z} \mid \mathbf{x})$ be given by $\mathcal{N}(\mathbf{z} ; h(\mathbf{x}), \mathbf{R})$, with a known measurement covariance matrix $\mathbf{R}$. The probability density of $t(\mathbf{z})$ given $\mathbf{x}$ can be approximated by linearizing $t$ :

Looking at equation (2), we can easily see that by definition $t \circ h$ can be linearized to

$$
t \circ h(\mathbf{x})=\mathbf{x}_{o}+\frac{\partial(t \circ h)}{\partial \mathbf{x}} \bar{K}\left(\overline{\mathbf{x}}_{o}-E\left[\overline{\mathbf{x}}_{o}\right]\right)=\mathbf{x}_{o}+G\left(\overline{\mathbf{x}}_{o}-E\left[\overline{\mathbf{x}}_{o}\right]\right)
$$

accordingly, we have

$$
p(t(\mathbf{z}) \mid \mathbf{x})=\mathcal{N}\left(t(\mathbf{z}) ; \mathbf{x}_{o}+G\left(\overline{\mathbf{x}}_{o}-E\left[\overline{\mathbf{x}}_{o}\right]\right), \frac{\partial t}{\partial \mathbf{z}} \mathbf{R} \frac{\partial t^{\prime}}{\partial \mathbf{z}}\right)
$$

and due to linearization we can switch $t(\mathbf{z})$ and $\mathbf{x}_{o}$,

$$
p\left(\mathbf{x}_{o} \mid \mathbf{z}, \overline{\mathbf{x}}_{o}\right)=\mathcal{N}\left(\mathbf{x}_{o} ; t(\mathbf{z})-G\left(\overline{\mathbf{x}}_{o}-E\left[\overline{\mathbf{x}}_{o}\right]\right), \frac{\partial t}{\partial \mathbf{z}} \mathbf{R} \frac{\partial t^{\prime}}{\partial \mathbf{z}}\right)
$$

where conditioning on $\mathbf{z}$ is the same as conditioning on $t(\mathbf{z})$. If we now substitute $\mathbf{x}=$ $K \mathbf{x}_{o}+\bar{K} \overline{\mathbf{x}}_{O}$

$$
\begin{aligned}
E\left[\mathbf{x} \mid \mathbf{z}, \overline{\mathbf{x}}_{o}\right] & =K t(\mathbf{z})+K G E\left[\overline{\mathbf{x}}_{o}\right]+(\bar{K}-K G) \overline{\mathbf{x}}_{o} \\
\operatorname{Cov}\left[\mathbf{x} \mid \mathbf{z}, \overline{\mathbf{x}}_{o}\right] & =K \frac{\partial t}{\partial \mathbf{z}} \mathbf{R} \frac{\partial t^{\prime}}{\partial \mathbf{z}} K^{\prime}
\end{aligned}
$$


To get rid of the conditioning on $\overline{\mathbf{x}}_{O}$ we go along the lines of Bayes total probability theorem for continuous random variables, averaging over $\overline{\mathbf{x}}_{o}$ we get,

$$
p(\mathbf{x} \mid \mathbf{z})=\int p\left(\mathbf{x} \mid \mathbf{z}, \overline{\mathbf{x}}_{o}\right) p\left(\overline{\mathbf{x}}_{o}\right) d \overline{\mathbf{x}}_{o} .
$$

Changing the conditioning and integrating, finally we get,

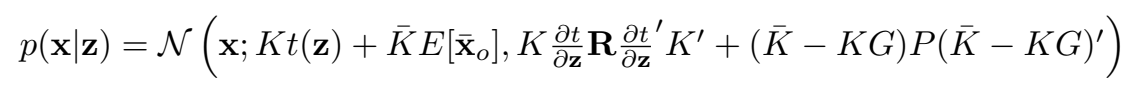

with the MAP (maximum a priori) estimator, $\hat{\mathbf{x}}=E[\mathbf{x} \mid \mathbf{z}]=K t(\mathbf{z})+\bar{K} E\left[\overline{\mathbf{x}}_{o}\right]$, and the covariance, $\mathbf{P}=\operatorname{Cov}[\mathbf{x} \mid \mathbf{z}]=K \frac{\partial t}{\partial \mathbf{z}} \mathbf{R} \frac{\partial t}{\partial \mathbf{z}}^{\prime} K^{\prime}+(\bar{K}-K G) P(\bar{K}-K G)^{\prime}$.

\section{Example: Track Initialization From Bearings-Only Measurements}

In this scenario only the spherical coordinates azimuth and elevation are measured, see Fig.(1). Initializing from $k$ measurements is possible, if the measurements are taken at different positions $\mathbf{x}_{s, i}$. Denoting $\mathbf{x}=[x, y, z, \dot{x}, \dot{y}, \dot{z}]^{\prime}=\left[\mathbf{x}_{o}, \overline{\mathbf{x}}_{o}\right]^{\prime}$ and $\mathbf{z}=[\phi, \theta]^{\prime}$, the measurement equations are the following,

$$
\phi=\arctan \left(\frac{y-y_{s}}{x-x_{s}}\right) \quad \theta=\arctan \left(\frac{z-z_{s}}{\sqrt{\left(x-x_{s}\right)^{2}+\left(y-y_{s}\right)^{2}}}\right) .
$$

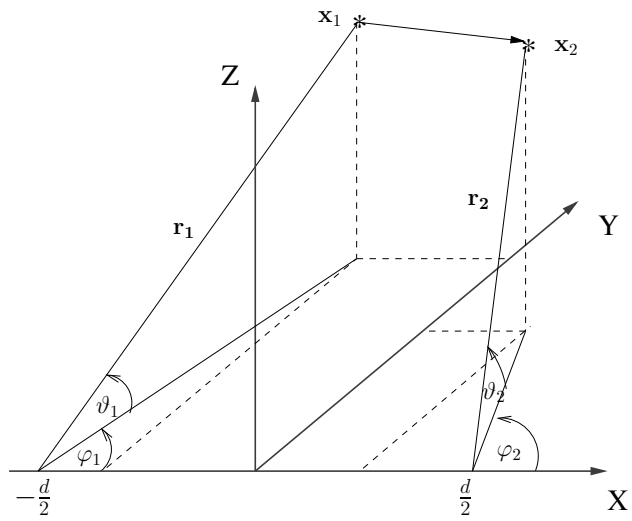

Figure 1: Bearings-only measurements scenario
To initialize the position $\mathbf{x}_{o}=[x, y, z]^{\prime}$ only, it is sufficient to have two measurements, i.e. $k=2$, which gives us $k \eta_{\mathbf{z}} \geq \eta_{\mathbf{x}_{o}}$. Those two measurements can be taken from two distinct arbitrary points $\mathbf{x}_{s, i}, i=1,2$ with distance $\left|\mathbf{x}_{s, 1}-\mathbf{x}_{s, 2}\right|=d$. W.l.o.g., we can assume them to be on the $x$-axis at $-d / 2$ and $d / 2$. In case of asynchronuous measurements, we need to model the velocities $\left(\overline{\mathbf{x}}_{o}=[\dot{x}, \dot{y}, \dot{z}]^{\prime}\right)$ to be zero mean Gaussian distributed, $\mathcal{N}\left(\overline{\mathbf{x}}_{o} ; 0, P\right)$, due to their impact on the measurements. As can be seen in Fig.(1), each pair of azimuth and elevation defines a ray originating at its measurement position $\mathbf{x}_{s}$.

Due to measurement errors and target velocity these rays will usually not intersect to render a valid solution. Considering only two azimuth and one elevation angles respectively, we will geometrically intersect a ray with a plane, which will generally be a well conditioned problem. Regarding two elevation and one azimuth angles, a cone replaces the plane; this problem is comparatively more likely to be ill conditioned. From these considerations it is 
easy to derive a mapping $t(\mathbf{z})$ according to Eq. (2), from which the mean and covariance can be calculated via the MAP estimator and covariance derived from Eq. (9).

Accordingly we can find four distinct solutions. After a one step Kalman filtering to merge the last datum, we select one solution using the trace of the estimated covariance matrix as an optimality criterion.

\section{Initializing via Unscented Transform}

To calculate $p(\mathbf{x} \mid \mathbf{z})$ in Eq. (9) we can replace the use of linearizations typical for the Extended Kalman filter, by using the Unscented Transform [JU04]. All we need for this is the functional relationship of the measurements $\mathbf{z}$, the unknown velocity and the position. Using $\mathbf{z}=h\left(\mathbf{x}_{o}, \overline{\mathbf{x}}_{o}\right)+\mathbf{w}$ we need to solve for $\mathbf{x}_{o}$, i.e. $\mathbf{x}_{o}=g\left(\mathbf{z}-\mathbf{w}, \overline{\mathbf{x}}_{o}\right)$. To find the probabilty density of $p\left(\mathbf{z}-\mathbf{w}, \overline{\mathbf{x}}_{o} \mid \mathbf{z}\right)$ we can use that $p(\mathbf{z}-\mathbf{w} \mid \mathbf{z})$ has obviously mean $\mathbf{z}$ and covariance $\mathbf{R}$ and $p\left(\overline{\mathbf{x}}_{o} \mid \mathbf{z}\right)$ is by modelling assumption $\mathcal{N}\left(\overline{\mathbf{x}}_{o} ; 0, P\right)$. Due to conditional independency we get $p\left(\mathbf{z}-\mathbf{w}, \overline{\mathbf{x}}_{o} \mid \mathbf{z}\right)=p(\mathbf{z}-\mathbf{w} \mid \mathbf{z}) p\left(\overline{\mathbf{x}}_{o} \mid \mathbf{z}\right)$ which we can transform into $p(\mathbf{x} \mid \mathbf{z})$, using $\mathbf{x}=K g\left(\mathbf{z}-\mathbf{w}, \overline{\mathbf{x}}_{o}\right)+\bar{K} \overline{\mathbf{x}}_{o}$.

Finding such a function $g$ is the major task in this approach. In the bearings only example above, this can be achieved again via geometrical considerations. Again, four different functions $g_{i}, i=1,2,3,4$ can be found by considering each triple of azimuth or elevation angles respectively and we will choose one by the minimum trace of the covariance, after merging the last datum via one step Kalman filtering.

\section{Numerical Results}

Using Monte-Carlo simulation, we generated numerical results for the bearings-only scenario. We chose the distance between the two sensors to be $d=10 \mathrm{~km}$ and generated random velocities according to our assumed pdf (uncorrelated random speeds in $x, y, z$ direction, $\sigma_{\dot{x}}=\sigma_{\dot{y}}=100 \mathrm{~m} / \mathrm{s}$ and $\sigma_{\dot{z}}=10 \mathrm{~m} / \mathrm{s}$ ), in a plane of $40 \mathrm{~km}$ by $40 \mathrm{~km}$ and constant height. The measurement noise has a standard deviation of $0.1^{\circ}$ in equally azimuth and elevation.

We plotted the results for 1000 Monte-Carlo runs for a height of $4 \mathrm{~km}$ and varying asynchronity $T$ between the two measurements in Fig. 2. Plot (a) shows the root-mean-square (RMS) position estimation error along the $x$-axis and a comparison with the Cramer-Rao lower bound calculated for $T=0$. We can see that the bound is achieved for $T=0$ and a constant increase of the RMS error for rising $T$. Plot (b) shows half of the symmetric plain for $T=2 \mathrm{~s}$, where the RMS error increases with distance from the sensors, but less so on the $y$-axis, which is orthogonal to the base spanned by the sensors.

In the same simulation we also checked the consistency of the covariance matrix by calculating the normalized estimation error squared (NEES). The results where always in the 95\% acceptance interval using the chi-square test, except for numerical difficulties with the Extended Kalman filter, if the target is right above one of the observers. 


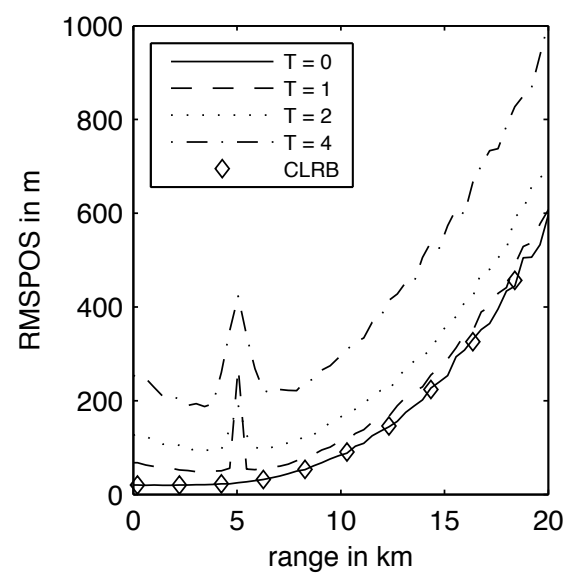

(a)

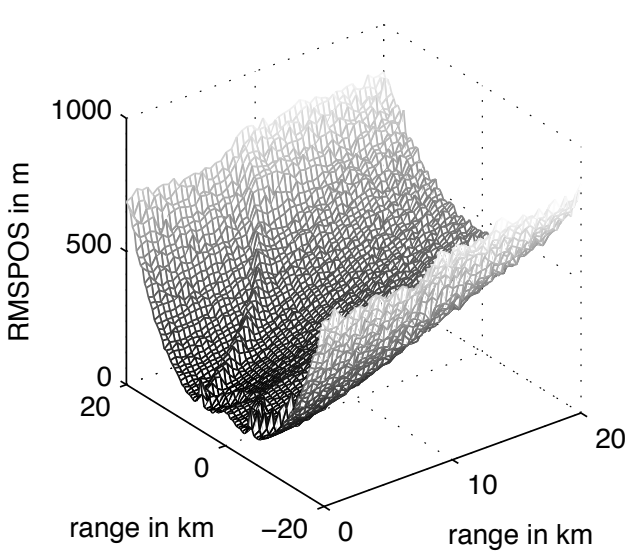

(b)

Figure 2: Plot (a) shows the RMS position error along the $x$-axis for different $T$; Plot (b) shows the RMS position error in a half plane for $T=2$

\section{Conclusion}

We have derived an analytical approach to initialize a generalized Kalman Filter from incomplete measurements, using a minimum number of measurements. Our derivation of the covariance matrix is adapted to model some components of the state vectors according to statistical assumptions. In an example using bearings-only measurements, we use geometrical considerations to implement our approach. We also consider using the Unscented Transform instead of linearizations. Both methods work well in this scenario. This work is largely based on an earlier report [vK91]. A more detailed study, which also considers the applicability of this method for other scenarios is in work and will be published as an FKIE research report soon.

\section{References}

[Bec00] Klaus Becker. Target Motion Analysis aus Winkelmessungen: Parametrische Studie in drei Dimensionen. FKIE Bericht 12, FGAN, Wachtberg-Werthoven, 2000.

[BSLK01] Y. Bar-Shalom, X. Rong Li, and T. Kirubarajan. Estimation with Application to Tracking and Navigation. Wiley-Interscience, 2001.

[JU04] Simon J. Julier and Jeffrey K. Uhlmann. Unscented Filtering and Nonlinear Estimation. Proceedings of the IEEE, 92(3):401-422, March 2004.

[KBSL01] T. Kirubarajan, Y. Bar-Shalom, and D. Lerro. Bearings-Only Tracking of Maneuvering Targets Using a Batch-Recursive Estimator. IEEE Trans. on Aerospace and Electronic Systems, 37(3):770-780, July 2001.

[vK91] G. van Keuk. Ein Basisalgorithmus für die räumliche Triangulation. FFM Bericht 418, FGAN, Wachtberg-Werthoven, 1991. 